\title{
Cerebral intraparenchymal extramedullary hematopoiesis in polycythemia vera
}

\author{
Jasmina Boban ${ }^{1,3} \cdot$ Peter Kalhs ${ }^{2} \cdot$ Majda M. Thurnher ${ }^{1}$
}

Received: 23 November 2018 / Accepted: 23 July 2019 / Published online: 1 August 2019

(c) The Author(s) 2019

Polycythemia vera (PV) is the most common myeloproliferative neoplasm, characterized by erythrocytosis, leukocytosis, and thrombocytosis and often associated with splenomegaly [1]. Neurological complications of PV are rare, but can include ischemic strokes, transient ischemic attacks (TIAs), and atypical TIAs [2].

Extramedullary hematopoiesis (EMH) is a compensatory physiological response of the organism to the failure of hematopoiesis at the bone marrow, which occurs commonly in patients with chronic hemolytic anemia or diseases with ineffective erythropoiesis [3]. Usually, extramedullary production of blood precursor cells occurs in the spleen, liver, or lymph nodes. However, cases with EMH in the adrenals, thymus, pleura, skin, gastrointestinal tract, and paranasal sinuses have been described [4]. Intracranial EMH is extremely rare, and only cases involving the dura mater have been described [3]. To the best of our knowledge, no cases of intracranial intraparenchymal EMH have been described.

A 48-year-old man with known polycythemia vera for 6 years presented with acute tinnitus. Six months before this, he was diagnosed with disease progression due to splenomegaly, anemia, leukocytosis $(100,000$ cells $/ \mu 1$,), and thrombocytopenia.

Magnetic resonance (MR) imaging of the brain was performed on a 1.5 T scanner (Philips Gyroscan Intera, NE), consisting of axial fluid attenuation inversion recovery (FLAIR), sagittal T1-weighted (T1WI), coronal T2-weighted

Majda M. Thurnher

majda.thurnher@meduniwien.ac.at

1 Department for Biomedical Imaging and Image-guided Therapy, University Hospital Vienna, Medical University of Vienna, Waehringer Guertel 18-20, 1090 Vienna, Austria

2 Department for Internal Medicine, University Hospital Vienna, Medical University of Vienna, Waehringer Guertel 18-20, 1090 Vienna, Austria

3 Present Address: Faculty of Medicine, University of Novi Sad, Hajduk Veljkova 3, Novi Sad 21000, Serbia
(T2WI) MR images, diffusion-weighted imaging (DWI), and a post-contrast T1WI study in two planes. In the right temporal lobe, there was a T1WI isointense (Fig. 1b) and T2WI hypointense (Fig. 1c), intraparenchymal 2-cm-lesion, surrounded by a rim of $\mathrm{T} 2 \mathrm{~W}$ high-intensity perilesional edema. The lesion was adjacent to the right temporal horn. On DWI, the lesion showed a low signal. On post-contrast T1W images (Fig. 1d), there was a patchy, dot-like enhancement. There were no other brain lesions.

Elective splenectomy was performed 2 days later, and histology revealed extramedullary hematopoiesis. On a follow-up brain MR scan, 6 weeks after the initial exam, the lesion showed a significant reduction in size, with central $\mathrm{T} 1 \mathrm{~W}$ - and $\mathrm{T} 2 \mathrm{~W}$ hyperintensity and a dark peripheral rim. No edema and no contrast enhancement were detected. Six weeks later, on the last follow-up MR scan, there was only a non-enhancing linear scar with hemosiderin deposits (Fig. 1e, f). The patient's neurological symptoms resolved completely. Three months after splenectomy, the patient underwent the first allogenic stem cell transplantation.

We present the first unique case of intracranial intraparenchymal EMH in a patient with polycythemia vera. PV is a myeloproliferative disorder, presenting with neurological complications mostly related to hypercoagulability and elevated hematocrit, such as ischemic strokes, TIAs, and atypical TIAs [2]. EMH is a compensatory, ectopic production of blood precursor cells in tissues other than bone marrow. Common sites of EMH are the liver, spleen, and lymph nodes. To date, cases of intracranial EMH have been described only in the form of extra-axial, epidural, or subdural, mass-like lesions [3]. The majority of the published cases were asymptomatic, presenting with symptoms only in cases of elevated intracranial pressure due to a tumor-like mass [5].

Imaging features of the lesions in our case were highly suggestive of extramedullary hematopoiesis; T1W isointensity and $\mathrm{T} 2 \mathrm{~W}$ hypointensity of the lesion were consistent with a high-cellularity lesion. Elevated diffusion 

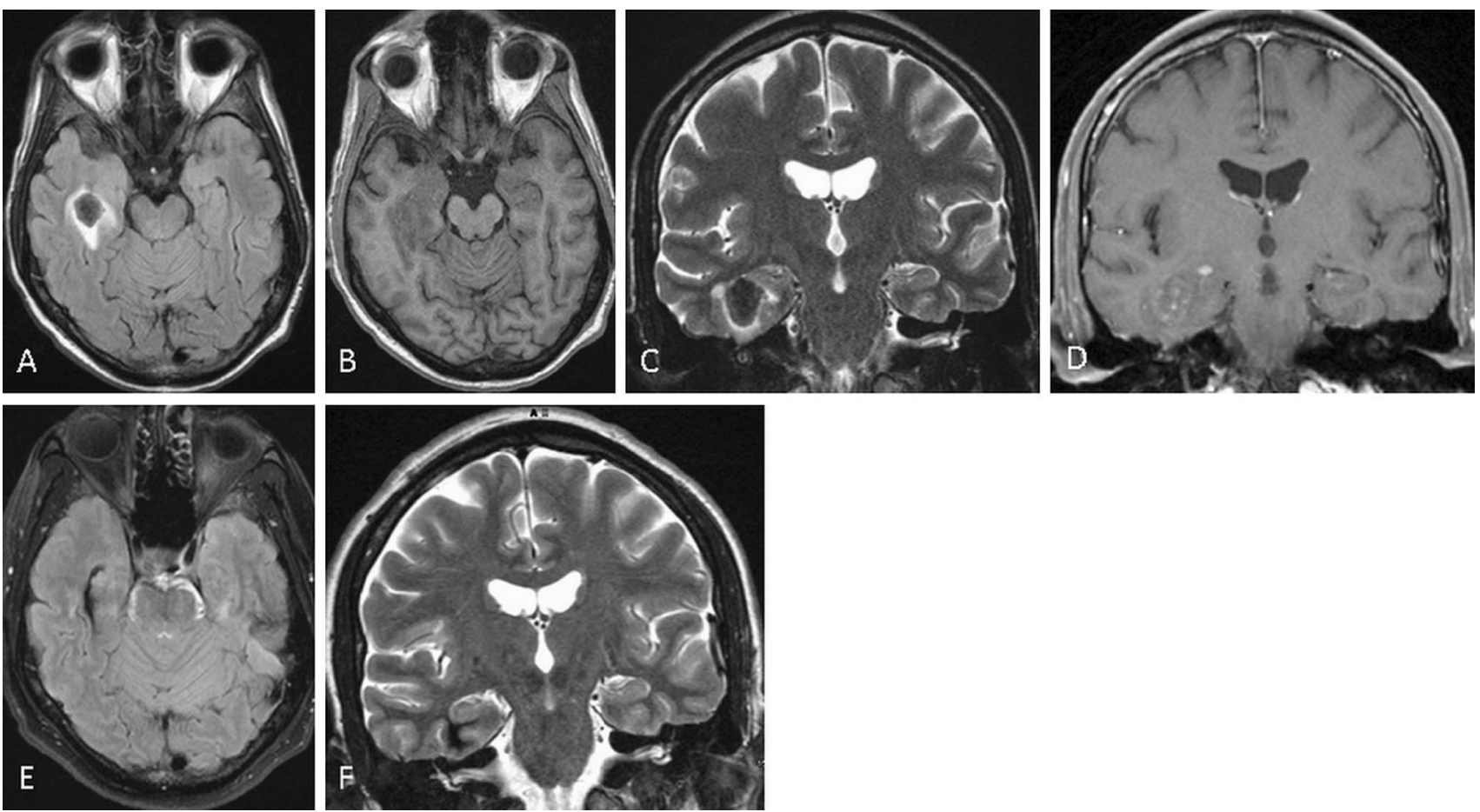

Fig. 1 On the initial MR exam, there was a 2-cm FLAIR hypointense (a), T1WI isointense (b), T2 hypointense (c) lesion in the right temporal lobe adjacent to the temporal horn, with perilesional edema and patchy contrast enhancement (d). At the 6-month follow-up MR scan, there was only a small scar with hemosiderin deposits $(\mathbf{e}, \mathbf{f})$

Author contributions JB drafted the manuscript and performed statistical analysis; PK was the attending physician and collected clinical data on the patient, and MMT analyzed MR images and critically revised the manuscript for intellectual content.

\section{Compliance with ethical standards}

Conflict of interest The authors declare that they have no conflict of interest.

Open Access This article is distributed under the terms of the Creative Commons Attribution 4.0 International License (http://creativeco mmons.org/licenses/by/4.0/), which permits unrestricted use, distribution, and reproduction in any medium, provided you give appropriate credit to the original author(s) and the source, provide a link to the Creative Commons license, and indicate if changes were made.

\section{References}

1. Spivak JL (2018) Polycythemia vera. Curr Treat Options Oncol 19(2): 12

2. Michiels JJ, Berneman Z, Schroyens W et al (2006) Platelet-mediated erythromelalgic, cerebral, ocular and coronary microvascular ischemic and thrombotic manifestations in patients with essential thrombocythemia and polycythemia vera: a distinct aspirinresponsive and coumadin-resistant arterial thrombophilia. Platelets 17(8):528-544
Acknowledgements Open access funding provided by Medical University of Vienna. 
3. Merchant R, Choudhari AJ, Verma M, Patkar DP, Doctor P (2018) Intracranial hematopoiesis in beta thalassemia: a case series. Ind J Pediatr 85(8):679-681

4. Ginzel AW, Kransdorf MJ, Peterson JJ, Garner HW, Murphey MD (2012) Mass-like extramedullary hematopoiesis: imaging features. Skelet Radiol 41:911-916

5. Eskazan AE, Ar MC, Baslar Z (2012) Intracranial extramedullary hematopoiesis in patients with thalassemia: a case report and review of the literature. Transfusion 52(8):1715-1720
6. Langguth B, Kreuzer PM, Kleinjung T, De Ridder D (2013) Tinnitus: causes and clinical management. Lancet Neurol 12:920-930

Publisher's Note Springer Nature remains neutral with regard to jurisdictional claims in published maps and institutional affiliations. 